\title{
INFLAMMATION AND GENES
}

\author{
Eliška Marklová \\ Charles University in Prague, Faculty of Medicine in Hradec Králové and University Hospital Hradec Králové, \\ Czech Republic: Department of Pediatrics
}

\begin{abstract}
Summary: Inflammation is a protective immune response to infection, trauma, or injury; however, only a subset of patients develops inflammation, suggesting other contributing factors involved, such as the environment and genes. Inflammationassociated genes involving those with pro- and anti-inflammatory effect should be properly balanced and regulated; the protein products of these genes ultimately determine the outcome of inflammation. Apart from gene mutations, gene polymorphisms related to some inflammatory markers also appear to correlate with the incidence and/or outcome of serious inflammatory events. Some genes recently recognized to be associated with inflammation are briefly reviewed. Modern genomic approaches, such as DNA micro-arrays and serial analysis of gene expression, allow for determining the extremely complex profile of inflammatory genes.
\end{abstract}

Abbreviations: A, adenine; AD, Alzheimer's disease; CARD15, caspase activating recruitment domain 15; C, cytosine; CCL26, chemokine ligand 26; CD, Crohn's disease; G, guanine; HF1/CFH, complement regulatory gene factor H; HLA, human leucocyte antigen; HP, Helicobacter pylori; IL, interleukin; MHC, major histocompatibility complex; NF- $\mathrm{B}$, nuclear factor kappa-B; NOD2, nucleotide-binding oligomerization domain; PLA2, phospholipase A2; PTPN22, protein tyrosine phosphatase, non-receptor type 22; RNAi, RNA interference; (SEPS1, selenoprotein S; SNPs, single-nucleotide polymorphisms; T, thymine; TNF, tumor necrosis factor; vacA, vacuolating cytotoxin

Key words: Inflammation; Genetic; Inflammation-associated genes; Polymorphisms

\section{Introduction}

Inflammation is the body's basic immune response to invasion of bacteria or viruses, and various other noxae; it is a protective mechanism to control damage, clean up the inducing agent and facilitate tissues reparation, followed by elimination of the inflammatory cells.

It would be wrong to equate infection and inflammation. There exist infectious diseases without inflammation, e.g., Creutzfeldt-Jacob disease, yellow fever, or opportunistic infections in AIDS. Non-infectious events that produce inflammation include trauma, radiation, physical or immunologic injury, tissue necrosis (except apoptosis), various poisonings, chemical or thermal burns, hypoxia, or reaction to one's own tissues. Within limits, inflammation is not itself a disease, but the process must be properly coordinated or phased, since any deficiencies or excess of the inflammatory response may result in a series of chain reactions causing morbidity and shortening lifespan $(29,41,42)$.

Almost every common disease involves an inflammation component. Chronic inflammation, affecting millions of people, in particular in the ageing, results in immuno- pathological changes that play a key role in the onset of degenerative diseases such as atherosclerosis, heart disease, chronic hepatitis, systemic lupus erythematosus, or rheumatoid arthritis, asthma, chronic inflammatory bowel disease, multiple sclerosis, Alzheimer's disease (AD), and also obesity and diabetes; it is even a major pathogenic factor for cancer $(1,21,22)$.

Various triggering conditions have been shown to activate inflammatory cells and induce a release of pro-inflammatory cytokines (interleukin (IL)-1, IL-17 and IL-18, tumor necrosis factor (TNF), and receptor activator of nuclear factor kappa-B ligand) from leukocytes, monocytes, and macrophages. This implies receptor aggregation, and subsequent activation of the nuclear factor kappa-B (NF$\kappa \mathrm{B})$ and the three major intracellular signaling pathways ERK, JNK and p38 MAPK (extracellular signal-regulated kinases, c-Jun N-terminal kinases, and p38 mitogen-activated protein kinases, respectively) $(16,40)$. An attribute of inflammation is a profound change in the gene expression of a large number of inflammatory proteins. Here various cytokines belong, including chemokines, cellular adhesion molecules, selectins, integrins, and metabolic enzymes (NADPH 
oxidase, phospholipase A2 (PLA2), cyclo-oxygenase-2, 5-lipoxygenase, inducible nitric oxide synthase, etc), which increase oxygen consumption and producing many oxygen-free radicals (36).

The etiology of many chronic inflammatory conditions is largely unknown. In fact, only a subset of patients develops inflammation, suggesting the presence of some contributing factors which may influence its onset: apart from the specific immune reactions that drive disease development, the environment also plays a role (diet, exposure to toxins, germs, pollutants, and inhalants), and so does genetics.

However, it is not clear to what extent genetic alterations interact with environmental variables to influence susceptibility to inflammation, or what the factors determining resolution and repair versus persistence and progression of inflammation are. Furthermore, there is little understanding of genetic factors that regulate compartmentalization of inflammation in one case, and engender a systemic response in another (27).

\section{Inflammation-associated genes}

Various genes are involved in inflammation; inflammatory stimuli signal to the nucleus to induce changes in the expression of a large number of genes whose protein products ultimately determine the outcome of inflammation.

Many clusters of genes studied represent expression profiles that correlate with the onset of inflammation and they are thus considered inflammation-associated genes. Some of the clusters contain genes that are not expressed physiologically ("early", or "late" inflammatory clusters), others contain genes that are commonly expressed at early stages of repair ("inflammation-maintained" clusters). Inflammation-associated genes and polypeptides encoded by those genes are either involved in the initiation and regulation of the inflammatory process (important pro-inflammatory IL-1, IL-6, IL-8, TNF and lipoteichoic acid), operate more distally in the cascade of inflammation (corticotrophin releasing hormone), or they may modify the process (C-reactive protein and protein C) (7).

An effective immune system requires rapid and appropriate activation of inflammatory mechanisms, but also equally rapid and effective resolution of the inflammatory state. Repressors of this process, called inflammation suppressor genes (e.g. those encoding IL-10, IL-4, and IL-13 cytokines) are also induced at each step of the activation. They represent proteins characterized by their induction as a response to the pathogen, typically late in the macrophage activation program; they include a class of proteins derived from alternate splicing of common signaling components.

Despite the many anti-inflammatory mechanisms available, there still exist a much larger number of genes implicated in chronic inflammatory diseases, which testifies to the unsteadiness of the whole system $(34,45)$.

Pro-inflammatory genes are commonly balanced by genes with an anti-inflammatory effect, thus constituting spe- cial pairs (e.g., IL-1 $\beta$ versus decoy receptors; xanthine oxidase versus superoxide dismutase; $\mathrm{Ca}^{2+}$-dependent $\mathrm{PLA}_{2}$ versus $\mathrm{Ca}^{2+}$-independent $\mathrm{PLA}_{2}$ ). Disregulation in the expression of pro- and anti-inflammatory genes is one of the milestones in inflammation development and progression.

Inflammation may be controlled by inhibiting many aspects of the inflammatory process through decreasing the transcription of inflammatory genes, and increasing the transcription of anti-inflammatory genes $(13,37)$.

\section{Gene mutations and polymorphism}

Mutations and gene polymorphisms are contributing factors in the pathogenesis of inflammation. A mutation is defined as any change in a DNA sequence away from normal, implying that a normal allele is prevalent in the population, and that the mutation changes this to a rare and abnormal form. Gene polymorphism means a variation in the DNA that is too common to be due merely to new mutation. Single nucleotide polymorphisms (SNPs, the most common genome sequence variation) occur when single base adenine (A), thymine (T), guanine $(G)$, and cytosine (C) substitutions involving nucleotides, building blocks of DNA, are replaced with others: e.g. A is replace with T; $\mathrm{A} \rightarrow \mathrm{T}$. Many SNPs are normal variations in the genome. Others are responsible for disease, and may affect how a person reacts to bacteria, viruses, drugs, and other substances.

Many studies have focused on mutations and polymorphisms that alter the primary sequence of proteins, resulting in functional variation (qualitative changes). However, affection of the steady-state level of mRNA molecules of a gene in a given cell (quantitative changes in gene expression) might also provide a significant source of variation (19). Interethnic and interindividual variability in the gene expression should also be considered. Recent reports indicate that the amount of copies of a particular gene (copy number variations, CNVs) contribute to nucleotide diversity, and possibly to genetic diseases, to a larger extent than SNPs (12).

Gene polymorphisms related to inflammatory markers (TNF, surfactant proteins, and Il-6), angiotensin converting enzyme, or pathogen receptors (monocyte differentiation surface antigen CD14, and the Toll-like, trans-membrane protein receptors) appear to correlate with the incidence and/or outcome of serious inflammatory events. This could have far-reaching implications due to its underlying role in serious chronic diseases $(2,30,33)$.

\section{Recent discoveries}

In recent years, some new genes have been identified as associated with inflammation.

\section{HLA-B27 associated inflammations}

The HLA genes encoded for by the loci on chromosome 6 (representing $6 \%$ of the human genome) are involved 
in antigen presentation. They are the most variable human genes, showing various polymorphic forms, one of which is the antigen B27 (the human major histocompatibility complex, MHC, located 6p21.3), a member of HLA-B group of cell surface molecules involved in immunity. Up to $20 \%$ of carriers have at least one of several associated conditions, including spondyloarthropathies, ankylosing spondylitis (Marie-Strumpell disease), inflammatory bowel disease, or isolated acute anterior uveitis (up to $50 \%$ of patients with the latter disease are HLA-B27 positive). The antigen is not causative however, and about $10 \%$ of normal subjects are B27-positive. Thus, since the association between HLA-B27 and disease is not absolute, other genes (non-B27 MHC genes, MHC-related genes, MHC genes involved in antigen processing and transplant, etc.) are likely to contribute to the susceptibility to those diseases $(28,43)$. Furthermore, HLA-B27 shares homology with a Klebsiella protein and may imply a bacterial pathogenesis (e.g. to ankylosing spondylitis) (24).

\section{Genes and blindness in the elderly}

Age-related macular degeneration is the most frequent cause of irreversible blindness in the elderly in developed countries. Genetic variations in the complement regulatory genes factor $\mathrm{H}$, when combined with a triggering event such as infection, may underlie the disorder breakout.

The major inhibitor of the alternative complement pathway factor $\mathrm{H}$ (immune response suppressor, encoded by the gene $\mathrm{HF} 1 / \mathrm{CFH}$ on chromosome 1q25-32) and NF-KB (transcription factor) predispose individuals with a variation in these genes to age-related macular degeneration (eight common HF1 SNPs have been found in $74 \%$ of all patients). In Caucasians, a polymorphism of the $\mathrm{C}$ allele of rs 1061170 (Y402H) in factor $\mathrm{H}$ was established as a susceptibility locus for an exudative type of the disease (18, 47).

\section{SEPS1 gene regulates inflammation in man}

Specific gene SEPS1 on chromosome 15q26.3 has recently been suggested as a candidate gene for traits related to inflammation such as diabetes mellitus and coronary heart disease. The physiological role of the encoded plasma membrane selenoprotein (SELS, SELENOS) in the endoplasmic reticulum is to regulate the red-ox balance and clear cells of misfolded proteins. Polymorphism in SEPS1 causes cumulation of these proteins, and under cell stress eventually leads to inflammation; carriers have higher IL-1 $\beta$, IL6, IL-10, and TNF concentrations. The SEPS1 has been considered a target gene for a specific RNA interference in the therapy (8).

\section{Tyrosine phosphatase gene and autoimmune diseases}

The protein product of the lymphoid protein tyrosine phosphatase gene PTPN22 (non-receptor type 22) located on human chromosome $1 \mathrm{p} 13.2$ is involved in preventing spontaneous T-cell activation (by dephosphorylating and inactivating T-cell receptor-associated c-src tyrosine kinase). A missense mutation of this gene (C1858T, R620W), found in $15-17 \%$ of the Caucasian population, was found to be associated with type 1 diabetes. Compelling evidence suggests that the PTPN22 is a common susceptibility gene for auto-immune diseases; significant association has been reported for rheumatoid arthritis, and systemic lupus erythematosus $(15,32,49)$.

\section{Filaggrin in eczema, asthma, and allergy}

The recent discovery of the gene that causes dry and scaly skin may change the eczema treatment approach. The human gene, located at $1 \mathrm{q} 21$, produces a protein called filaggrin (filament aggregating protein) $(3,35)$, which helps to keep the skin protected by forming an outer barrier and keeping the water in. In healthy people, this protein is abundant, but in absence caused by genetic variants (R510X and $2282 \mathrm{del})$ the skin dries out and begins to flake off. Reportedly, about $60 \%$ of Danish children carrying filaggrin mutation get eczema in the first 2 years of life; according to another study, two thirds of Irish children with eczema carry this mutation. A very strong link between filaggrin mutation and eczema was found among Scottish children with asthma (38).

\section{Genes linked to disorder of the oesophagus}

The eotaxin gene family recruits and activates eosinophils, basophiles and Th2 lymphocytes that play a major role in allergic disorders. Polymorphism of the eotaxin-3 gene (chemokine ligand 26, CCL26, located on chromosome 7q11.23) coding for a small inducible cytokine A26 precursor triggers a harmful, possibly allergic-based inflammation. The disorder, called eosinophilic oesophagitis, presents in children and adults with failure to thrive, painful swallowing and digestion, vomiting, and weight loss. This new disease, with a genetic predisposition and accelerating incidence (1:10 000) leading to ulcers and cancer, may be confused (without biopsy) with heartburn or acid reflux $(5,14)$.

\section{Crohn's disease (CD)}

$\mathrm{CD}$ is a disorder characterized by intestinal and systemic chronic inflammation (incidence 1-10/100 000) with typical relapses/remissions occurring in adolescence or early adulthood.

The pathogenesis of CD is complex and appears to involve many factors. Commonly classified as an immune-mediated tissue injury, $\mathrm{CD}$ is considered to be a consequence of a delayed-type hypersensitivity reaction due to a failure to establish tolerance (to food antigens), or a breakdown in tolerance (to own gut bacteria). However, other factors also seem to be important in the CD pathogenesis: environmental factors such as cigarette smoking, triggering the inflammatory response, and distinct genetic components.

The gene called NOD2 (nucleotide-binding oligomerization domain 2, on chromosome 16q12) has been identi- 
fied as a locus for genetic susceptibility in CD. The NOD2, recently renamed as caspase activating recruitment domain 15 (CARD15), belongs to a family of NOD1/apoptotic protease activating factor 1 related proteins which regulate apoptosis and some of which activate NF- $\mathrm{KB}$ ). Variants of the gene (namely the NOD2 mutant 3020insC, showing an impaired ability to activate $\mathrm{NF}-\mathrm{\kappa B}$ ) have been associated with a risk for $\mathrm{CD}$; they account for about $25 \%$ of the $\mathrm{CD}$ patients $(6,31,44)$.

Decreased expression of the (cell death regulatory) protein GRIM-19, which interacts with NOD2 and serves as an effector of anti-bacterial function in intestinal epithelial cells, is supposed to play a key role in CD pathogenesis (4).

Quite recently, a highly significant association between $\mathrm{CD}$ and the IL23R gene on chromosome $1 \mathrm{p} 31$ has been found. This gene, encoding a subunit of the receptor for the proinflammatory cytokine IL-23, has long been linked to inflammatory bowel diseases and auto-immune psoriasis. An uncommon coding variant (rs11209026, c.1142G $\rightarrow$ A, p.Arg381Gln) confers strong protection against $\mathrm{CD}$, and additional non-coding IL23R variants are independently associated. This signaling pathway is supposed to be a better therapeutic target in inflammatory bowel disease than CARD15/NOD2 (10).

\section{Helicobacter pylori $(\mathrm{HP})$ and related genes}

$H P$ infection in the human stomach is rather common, causing gastritis, peptic ulcer disease and gastric malignancy (also associated with coronary disease); infection is usually acquired in childhood and persists throughout life. $H P$ synthesizes an unusual GroES homolog (bacterial co-chaperonin, heat shock gene product of Escherichia coli), a protein-folding factor, which stimulates the production of IL-8, IL-6, and others. Consequently, an altered expression of the Bcl-2, Bax, and c-Myc genes induces morphological changes in gastric epithelial cells, typical of apoptosis. Immune response mediators have also been suggested as one possible explanation for the observed association between $H P$ and several respiratory, as well as cardiovascular, rheumatic, skin and liver pathologies $(11,20,25,46)$. The IL-8 A251A $\rightarrow$ T (rs4073) variant, and the polymorphisms in $H P$ vacA (vacuolating cytotoxin) gene may also be involved in limiting the infection outcome to gastritis, peptic ulcer or cancer onset (17).

\section{Alzheimer's disease (AD)}

There is increasing evidence of a relationship between inflammation factors and dementia in the elderly people. Previous research in $\mathrm{AD}$ has focused on a protein called beta-amyloid. Later on, mutations in the tau gene (on chromosome 17) encoding tau- (HN9, a microtubule-binding) protein, have been found in patients; many other neurodegenerative disorders have also tau abnormalities ("tauopathies"). The risk of AD is furthermore substantially influenced by at least 10 different polymorphisms in the in- flammatory agents IL-1 $\beta$, IL-6, TNF, $\alpha 2$-macroglobulin, and $\alpha 1$-antichymotrypsin. The causal interpretation of a relationship is supported by a strong increase in the number of inflammation markers many (up to 25) years before dementia develops, as has been found in the children of patients with $\mathrm{AD}(23,39)$.

Recent discoveries in genetics have not only increased our knowledge of etiology and pathogenesis of diseases, but also opened new possibilities for therapeutic intervention.

\section{Inflammation and RNAi}

New technologies such as RNA interference (RNAi) are used to knock down the target protein expression levels in mammalian cells, and might be used in therapy. RNAi (2006-Nobel Prize estimated discovery) (9) is a method based on a specific suppression of genes by synthetic, short double-stranded interfering RNA sequence that is complementary to the mRNA transcribed from the target gene. The interference would block the production of an encoded protein. An example is presented for illustration: Many inflammatory genes are regulated by $\kappa \mathrm{B}$ sites; NF- $\kappa \mathrm{B}$ mediated inflammation is a key process in many diseases. Application of the synthetic p65 subunit of NF- $\mathrm{kB}$ leading to low production of the p65 protein and IL- 8 secretion suppression is a useful tool for efficient gene silencing. Also, the p50 subunit of NF- $\kappa B$ was found to suppress the expression of $\kappa \mathrm{B}$-associated genes, thus preventing inflammatory injury during Escherichia coli pneumonia, a common infection in hospitals $(26,48)$.

\section{Conclusions}

Inflammatory diseases represent a close ground of gene expression, which might be extremely complex owing to the diversity of cell types involved in their pathology. There is apparent progress in the field, with hundreds of new gene variants discovered and characterized; however, firm evidence consistently linking them with pathogenesis of complex chronic diseases is still limited. To find true causes and evaluate appropriate treatments will require time-consuming resolution of specific variations in inflammatory gene expression.

The genotyping of common mutations is carried out by restriction fragment length polymorphism and amplification refractory mutation system techniques. Serial analysis of gene expression (SAGE), differential display techniques, and both cDNA and oligonucleotide array-based technologies have been applied for genome-wide gene expression exploring. Such an approach facilitates 1) detection of previously unsuspected genes with a likely role in inflammation, 2) follow-up of much over- and underexpression of gene populations compared with controls, and 3) identification of phenotypically associated specific patterns of gene expression. 


\section{Acknowledgements}

Contributed as a lecture to the CEEPUS Summer University on Inflammation, Multidisciplinary Approach, 2006, Zadar, Croatia.

Supported by the Research Project MZO 00179906.

\section{References}

1. Adarichev VA, Vermes C, Hanyecz A, Mikecz K, Bremer EG, Glant TT. Gene expression profiling in murine autoimmune arthritis during the initiation and progression of joint inflammation. Arthr Res Ther 2005;7: R196-207.

2. Arcaroli J, Fessler MB, Abraham E. Genetic polymorphisms and sepsis. Shock $2005 ; 24: 300-12$

3. Barker JN, Palmer CN, Zhao Y, et al. Null mutations in the filaggrin gene (FLG) determine major susceptibility to early-onset atopic dermatitis that persists into adulthood. J Invest Dermatol. 2006 Sep 21; [Epub ahead of print].

4. Barnich N, Aguirre JE, Reinecker HC, et al. Membrane recruitment of NOD2 in intestinal epithelial cells is essential for nuclear factor-kB activation in muramyl dipeptide recognition. J Cell Biol 2005;170:21-6.

5. Blanchard C, Wang N, Stringer KF, et al. Eotaxin-3 and a uniquely conserved gene-expression profile in eosinophilic esophagitis. J Clin Invest 2006;116:536-47.

6. Buhner S, Buning C, Genschel J, et al. Genetic basis for increased intestinal permeability in families with Crohn's disease: role of CARD15 3020insC mutation? Gut 2006;55:342-7.

7. Cooper L, Johnson C, Burslem F, Martin P. Wound healing and inflammation genes revealed by array analysis of 'macrophageless' PU.1 null mice. Genome Biology 2004,6:R5

8. Curran JE, Jowett, JBM, Elliott KS et al. Genetic variation in selenoprotein $\mathrm{S}$ influences inflammatory response. Nat Genet 2005;37:1234-41.

9. Daneholt B. Advanced Information. RNA interference. The Nobel Prize in Physiol or Med 2006. http://nobelprize.org/nobel_prizes/medicine/laureates/2006/adv.html

10. Duerr RH, Taylor KD, Brant SR, et al. A genome-wide association study identifies IL23R as an inflammatory bowel disease gene. Science 2006;314:1461-3.

11. Ferrero RL, Thiberge M, Kansau I, et al. The GroES homolog of Helicobacter py lori confers protective immunity against mucosal infection in mice. Proc Natl Acad Sci U S A. 1995;92:6499-503.

12. Freeman JL, Perry GH, Feuk L, et al. Copy number variation: new insights in genome diversity. Genome Res. 2006;16:949-61.

13. Goodwin JS. Are prostaglandins proinflammatory, antiinflammatory, both or neither? J Rheumatol 1991;18 (Supp28):26-9.

14. Gupta SK, Fitzgerald JF, Kondratyuk T, Hogenesch H. Cytokine expression in normal and inflamed esophageal mucosa: A study into the pathogenesis of allergic eosinophilic esophagitis. J Pediatr Gastroenterol Nutr 2006;42:22-6.

15. Hinks A, Worthington J, Thomson W. The association of PTPN22 with rheumatoid arthritis and juvenile idiopathic arthritis. Rheumatology 2006;45:365-8.

16. Johnson GL, Lapadat R. Mitogen-Activated Protein Kinase Pathways Mediated by ERK, JNK, and p38 Protein Kinases. Science 2002;298:1911-12.

17. Kamali-Sarvestani E, Bazargani A, Masoudian M, Lankarani K, Taghavi A-R, Saberifiroozi M. Association of $H$ pylori cagA and vacA genotypes and IL-8 gene polymorphisms with clinical outcome of infection in Iranian patients with gastrointestinal diseases. World J Gastroenterol 2006;12:5205-10.

18. Klein RJ, Zeiss C, Chew EY, et al. Complement factor $\mathrm{H}$ polymorphism in agerelated macular degeneration. Science 2005;308:362-4.

19. Korstanje R, Paigen B. From QTL to gene: the harvest begins. Nat Genet 2002;31:235-6.

20. Lin Y-F, Wu M-S, Chang CC, et al. Comparative immunoproteomics of identification and characterization virulence factors from Helicobacter pylori related to gastric cancer. Mol Cell Proteomics 2006;5:1484-96.

21. Mattson MP, Camandola S. NF-kB in neuronal plasticity and neurodegenerative disorders. J Clin Invest 2001;107:247-54.
22. McGeer PL, McGeer EG. Inflammation and the degenerative diseases of aging. Annals NYAS 2004:1035:104-6.

23. McGeer PL, McGeer EG. Polymorphisms in inflammatory genes and the risk of Alzheimer disease. Arch Neurol 2001;58:1790-2.

24. Mehra NK, Kanga U. Molecular diversity of the HLA-B27 gene and its association with disease. J Modern Rheumatol 2001;11:275-285.

25. Meyer-ter-Vehn T, Covacci A, Kist M, Pahl HL. Helicobacter pylori activates MAP kinase cascades and induces expression of the proto-oncogenes c-fos and c-jun. J Biol Chem 2006;275:16064-72.

26. Mizgerd JP, Lupa MM, Spieker MS. NF-kB p50 facilitates neutrophil accumulation during LPS-induced pulmonary inflammation. BMC Immunology 2004;5: $1-11$.

27. Munford RS, Pugin J. Normal responses to injury prevent systemic inflammation and can be immunosuppressive. Am J Respir Crit Care Med 2001;163:316-21.

28. Murray PI, Sivaraj RR. Anti-TNF- therapy for uveitis: Behcet and beyond. Eye 2005; 19:831-3.

29. Nathan C. Points of control in inflammation. Nature 2002;420:846-52.

30. Nieters A, Beckmann L, Deeg E, Becker N. Gene polymorphisms in Toll-like receptors, interleukin-10, and interleukin-10 receptor alpha and lymphoma risk. Genes Immun 2006;7:615-24

31. Ogura Y, Bonen DK, Inohara N, et al. A frameshift mutation in NOD2 associated with susceptibility to Crohn's disease. Nature 2001;411:603-6.

32. Onengut-Gumuscu S, Buckner JH, Concannon P. A Haplotype-based analysis of the PTPN22 locus in type 1 diabetes. Diabetes 2006;55:2883-89.

33. Pena AS. Genetics of inflammatory bowel disease. The candidate gene approach susceptibility versus disease heterogeneity. Dig Dis 1998;16:356-63.

34. Phillips K, Kedersha N, Shen L, Blackshear PJ, Anderson P. Arthritis suppressor genes TIA-1 and TTP dampen the expression of tumor necrosis factor, cyclooxygenase 2, and inflammatory arthritis. Proc Natl Acad Sci USA 2004;101:2011-16.

35. Rothnagel JA, Steinert PM. The structure of the gene for mouse filaggrin and a comparison of the repeating units. J Biol Chem 1990;265:1862-5.

36. Saklatvala J, Dean J, Clark A. Control of the expression of inflammatory response genes. Biochem Soc Symp 2003;70:95-106.

37. Scuderi $\mathrm{F}$, Convertino R, Molino N, et al. Effect of pro-inflammatory/anti-inflammatory agents on cytokine secretion by peripheral blood mononuclear cells in rheumatoid arthritis and systemic lupus erythematosus. Autoimmunity 2003;36:71-7.

38. Smith FJ, Irvine AD, Terron-Kwiatkowski A, et al. Loss-of-function mutations in the gene encoding filaggrin cause ichthyosis vulgaris Nature Genetics 2006; $38: 337-42$.

39. Swanberg M, Duvefelt K, Diez M, et al. Genetically determined susceptibility to neurodegeneration is associated with increased expression of inflammatory genes. Neurobiol Dis 2006;24:67-88.

40. Tak PP, Firestein GS. NF-kB: a key role in inflammatory diseases. J Clin Invest 2001;107:7-11.

41. Tracey KJ, Warren HS. Human Genetics: An inflammatory issue. Nature 2004; 429:35-7.

42. Tracey KJ. The inflammatory reflex. Nature 2002;420:853-85.

43. Waheed NK, Miserocchi E, Foster CS, et al. Ocular concerns in juvenile rheumatoid arthritis. Immunol Inflamm. Ocul Immunol Inflamm. 2005;13:205-12.

44. Wehkamp J, Stange EF. A new look at Crohn's disease: breakdown of the mucosal antibacterial defense. Ann NY Acad Sci 2006;1072:321-31.

45. Wells CA, Ravasi T, Hume DA. Inflammation suppressor genes: please switch out all the lights. J Leukoc Biol 2005;78:9-13.

46. Yang Y, Deng CS, Peng JZ, Wong BC-Y, Lam SK, Xia HH-X. Effect of Helicobacter pylori on apoptosis and apoptosis related genes in gastric cancer cells. Mo Pathol 2003;56:19-24.

47. Zareparsi S, Kari E, Branham KEH, Li M, et al. Strong association of the $\mathrm{Y} 402 \mathrm{H}$ variant in complement factor $\mathrm{H}$ at $1 \mathrm{q} 32$ with susceptibility to age-related macular degeneration. Am J Hum Genet 2005;77:149-53.

48. Zhang J. Dampening the silencing effect of RNA interference in mammals. Biochem J 2005;390:675-9.

49. Zheng W, She J-X. Genetic association between a lymphoid tyrosine phosphatase (PTPN22) and type I diabetes. Diabetes 2005;54:906-8.

Submitted December 2006. Accepted February 2007.

\section{Corresponding author:}

Eliška Marklová, M.D., University Hospital Hradec Králové, Department of Pediatrics, Sokolská 581, 50005 Hradec Králové, Czech Republic, e-mail: marklova@lfhk.cuni.cz 